\title{
Effect of Automatic Ball Balancer on Unbalanced Rotor Vibration
}

\author{
$\left\{\text { Michael Makram*, Ahmed F. Nemnem }{ }^{\dagger} \text {, and Mohamed K. Khalil }\right\}^{\S}$
}

\begin{abstract}
Rotor vibration due to unbalance causes a lot of problems during operation. One of the simplest ways to reduce rotor vibration is the passive balancing. Unbalanced vertical rotor is modeled by a two degrees of freedom system considering Jeffcott model assumption. A $(2+n)$ degrees of freedom mathematical model is derived with respect to a Cartesian co-ordinate system for the unbalanced rotor with the automatic ball balancer. The model equations are expressed as state equations then solved numerically to calculate vibration amplitudes and angular positions of balancing balls. Model validation is achieved by comparing these results with a three-dimensional model. The effect of the automatic ball balancer on vibration amplitudes is explained at different speed ranges. A parametric study is done to explain the effect of the system damping coefficient on the ball balancer behaviors.
\end{abstract}

Keywords: Rotor vibrations, unbalance, online balancing, passive balancing, automatic ball balancer, dynamic balancer.

\section{Nomenclature}

A vibration amplitude.

a radial distance between ball center and disk center.

c damping coefficient.

$\mathrm{c}_{\mathrm{b}} \quad$ damping coefficient for balancing ball motion in oil filled track.

e Eccentricity

i order of ball.

$\mathrm{k} \quad$ shaft stiffness.

$\mathrm{m} \quad$ mass of disk.

$\mathrm{m}_{\mathrm{b}} \quad$ mass of balancing ball in one ball $\mathrm{ABB}$.

$\mathrm{m}_{\mathrm{b} 1}, \mathrm{~m}_{\mathrm{b} 2}$ mass of balancing ball in two balls ABB.

$\mathrm{m}_{\mathrm{imb}} \quad$ rotor imbalance mass.

$\mathrm{n} \quad$ number of balancing balls.

$\mathrm{t}$ time.

$\mathrm{x} \quad$ linear displacement in $\mathrm{x}$-axis direction.

$\mathrm{y} \quad$ linear displacement in $\mathrm{y}$-axis direction.

$\theta \quad$ angular displacement of disk.

$\theta_{\mathrm{b}} \quad$ angular displacement of ball.

$\omega \quad$ angular velocity of rotor.

$\begin{array}{ll}* & \text { Michael@mtc.edu.eg } \\ + & \text { Farid_nemnem@mtc.edu.eg } \\ \S & \text { M_khalil@mtc.edu.eg } \\ \text { Egyptian Armed Forces, Egypt. }\end{array}$




\section{Abbreviations}

ABB automatic ball balancer.

DOF degree of freedom.

\section{Introduction}

Rotors are commonly used in several systems including vehicle wheels, electrical motors, machine tools, compressors, turbo machinery, aircraft gas turbine engines and helicopter blades. Vibration affects all these rotating systems. Unbalance, misalignment, bowed shafts, material imperfections and cracks are some causes of the vibration, but mass imbalance still one of the most primary sources of vibrations, which occurs when the principal inertia axis of the rotor is not coincident with rotational axis. An imbalance can arise through imperfections in the manufacturing process or resulting from wear, missing balance weights or damage. A heavy spot in a rotating component will cause vibration when the unbalanced weight rotates around the rotor axis, creating a centrifugal force. So, that imbalances can create the worst operating conditions by causing translational and rotational vibrations which in turn cause large stresses. As rotor speed changes, the effects of imbalance may become higher. In some cases, imbalances can be factors in poor performance, high noise levels, reduced bearing life, and reduced human comfort.

Balancing methods can be classified to two common balancing methods as shown in Fig. 1, one method is off-line balancing in which the rotating machine is stopped for the adjustment of mass distribution, and the second method is on-line balancing in which the mass distribution rearrangement happens continuously during rotation. Because it is often impractical to take a machine out of service to realign and balance its components, especially if balancing usually needs to be done, engineers have long searched for ways to automatically balance equipment using simple devices that allow mass redistribution to compensate for any unknown imbalance.

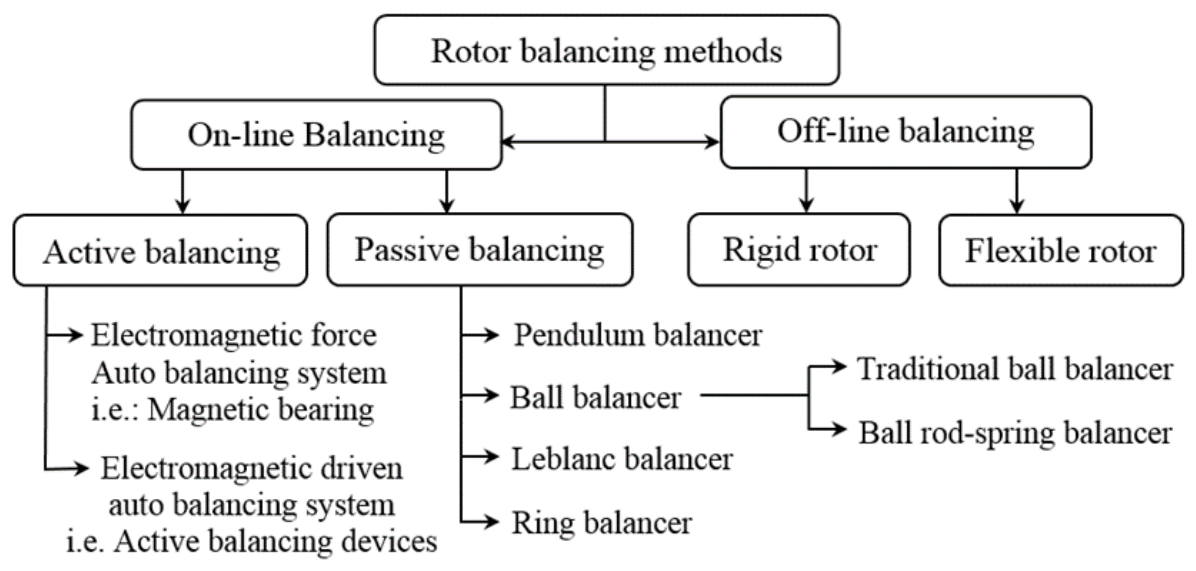

Fig. 1 Balancing methods classification

There are two types of automatic balancers, active and passive. Active devices utilize computers and sensors which continuously read the vibrations, then apply control laws to counteract these vibrations and most of them use electromagnetic actuators and can balance the machine very effectively [1], but from its disadvantages are complexity, expensive cost and high weight. Where the passive balancers usually consist of freely moving masses, tend to move to positions that counteract the inherent imbalance of the system and do so without the use of computers and control laws. Simplicity, reliability, and relatively low cost of passive balancing systems make them a very attractive solution, and thus they have been significant subjects for past research. Applications of automatic balancers (ABs) include optical disk drives, jet engines, internal-combustion engines, washing machines, food blenders, grinding wheels, and vehicle wheels. 
The ring balancer was the first form of automatic balancers that appeared in 1872 by A. Fesca who patented improved centrifugal machine equipped with three rings balancer [2], in this balancer the freely moving masses are rings. In the same year, the second type of passive balancers was registered by $\mathrm{M}$. Leblanc who patented his automatic balancer for rotating bodies, in this design the balancer consisted of a simple cylindrical chamber field with a heavy liquid [5] and it was called Lablanc balancer. The third type is the ball balancer which was first designed in a detailed experimental study by Thearle [3] in 1932. In $1946 \mathrm{~K}$. Clark used four non-centrally attached pendulums to reduce the level of centrifugal machines' vibrations, and this is considered the first documentation of pendulum type balancer [4]. Thearle [5] compared several different types of automatic dynamic balancers, such as a ring, pendulum and ball balancers. This is the first appearance of an automatic pendulum-balancer in the literature. In this paper Thearle concluded that by placing the pivot of pendulums at the center of rotation the pendulum balancer become equivalent to the ring balancer. In addition, he concluded that ball balancers were a superior system.

Passive balancing techniques especially pendulum and ball automatic balancers have received a great deal of attention in recent research. Automatic passive self-balancing systems are important tools for reducing the effects of synchronous vibration in a variety of rotating machinery. Such systems are capable of precise balancing at certain speed ranges above the critical speed which is called stable region, but they may play an opposite role in other speeds. Pendulum balancers tend to be costlier to construct than ball balancers and the weight of the pendulums must be supported in special ways, which leads to additional mechanical complexity, however, ball balancer systems have some problems such as friction between the balls and the cylindrical race or channel where they move, the ball balancer still more popular than the pendulum balancer and successfully applied to different fields. A traditional ball-type balancer is composed of several balls moving only in tangential direction along a fixed circular orbit. To increase the stable region of the perfect balancing configuration, a new design of balancer was proposed [ 6 ] in which an extra degree of freedom in the radial direction is introduced to the balls.

\section{Mathematical Model}

The rotor shaft system is considered as a 2 DOF system taking in consideration Jeffcott model assumptions. The used model as shown in Fig. 2 consists of: a vertical simply-supported massless flexible shaft, and a disk mounted at the mid span of the shaft is rotating in a horizontal plane with a radial mass imbalance causing a shift between its geometry center and its center of gravity. The disk model has two degrees of freedom, $\mathrm{x}$ and $\mathrm{y}$, which are mutually orthogonal linear displacements in the same horizontal plane. The model is symmetric, having the same spring stiffness $\mathrm{k}_{\mathrm{x}}, \mathrm{k}_{\mathrm{y}}$ and damping coefficient $\mathrm{c}_{\mathrm{x}}, \mathrm{c}_{\mathrm{y}}$ in both directions, and ideal friction bearing is assumed.

The differential equations of motion

$$
\begin{gathered}
m \ddot{x}+c \dot{x}+k x=-m e \omega^{2} \cos \omega t \\
m \ddot{y}+c \dot{y}+k y=-m e \omega^{2} \sin \omega t \\
c=c_{x}=c_{y}, k=k_{x}=k_{y}
\end{gathered}
$$

The analytical solution of radially unbalanced system is: 


$$
\begin{gathered}
x(t)=\frac{\omega^{2} e}{\sqrt{\left(\frac{k}{m}-\omega^{2}\right)^{2}+\left(\frac{c \omega}{m}\right)^{2}}} \cos (\omega t-\phi) \\
y(t)=\frac{\omega^{2} e}{\sqrt{\left(\frac{k}{m}-\omega^{2}\right)^{2}+\left(\frac{c \omega}{m}\right)^{2}}} \sin (\omega t-\phi) \\
A=\sqrt{x^{2}+y^{2}}
\end{gathered}
$$

Each ball in the ABB reaches its own angular coordinate $(\theta)$, and this coordinate is independent on the linear coordinates of the rotor, so that the rotor and the ABB can be modeled by a $(2+n)$ DOF system, where (n) is the number of balls in ABB.

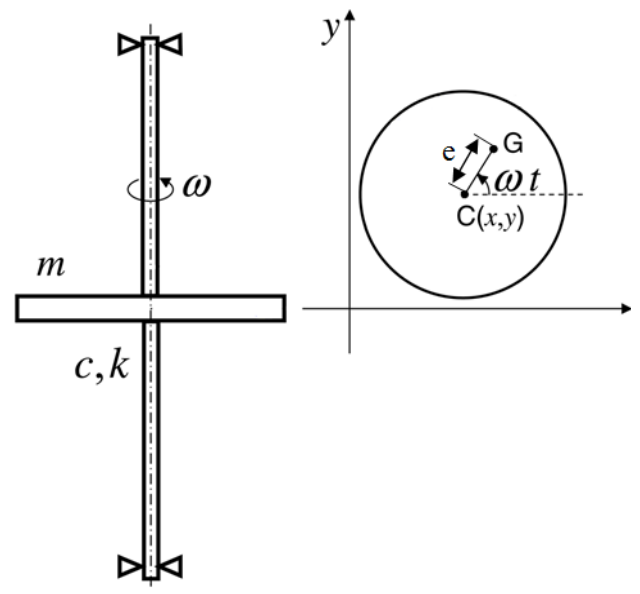

Fig. 2 Jeffcott model

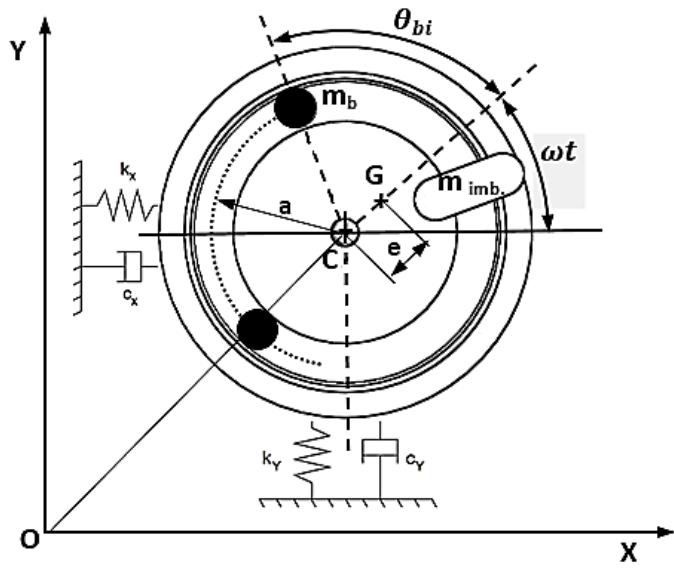

Fig. 3 Schematic drawing of an automatic dynamic balancer

An eccentric rotating disc is studied with an $\mathrm{ABB}$ consisting of several balls free to move in a race filled with a viscous fluid and positioned at a fixed distance from the center of rotation of the disc. This set-up is shown schematically in Fig. 3. Point (G) represents the center of mass of the disc (without the balancing balls) and is located a distance (e) from the center of rotation $(\mathrm{C})$.

The equations of motion of our model are derived, where the assumption that all motion is confined to the two-dimensional plane. Also, it is assumed that no interactions between the balls. This assumption is valid provided the balls are in an equilibrium state. Note that neglecting impacts between the balls makes this study similar to that of an ABB with double ball races [7].

$$
\begin{aligned}
& m \ddot{x}+c \dot{x}+k x=-m e \omega^{2} \cos \omega t-\sum_{n=1}^{i} m_{b n} a\left[\ddot{\theta}_{b n} \sin \left(\omega t+\theta_{b n}\right)+\left(\omega+\dot{\theta}_{b n}\right)^{2} \cos \left(\omega t+\theta_{b n}\right)\right] \\
& m \ddot{y}+c \dot{y}+k y=-m e \omega^{2} \sin \omega t+\sum_{n=1}^{i} m_{b n} a\left[\ddot{\theta}_{b n} \cos \left(\omega t+\theta_{b n}\right)-\left(\omega+\dot{\theta}_{b n}\right)^{2} \sin \left(\omega t+\theta_{b n}\right)\right] \\
& m_{b n} a^{2} \ddot{\theta}_{b n}+c_{b n} a^{2} \dot{\theta}_{b n}=-m_{b n} a\left[\ddot{x} \sin \left(\omega t+\theta_{b n}\right)-\ddot{y} \cos \left(\omega t+\theta_{b n}\right)\right]
\end{aligned}
$$


Equations (6) and (7) describe the horizontal and vertical displacements in the same plane, equation (8) is the equilibrium equation of ball in the tangential direction, and for more than one ball $\mathrm{ABB}$ this equation is repeated for each ball.

\section{Numerical Solution}

The state equations can be conveniently used in solving the model equations, and these equations are solved numerically using MATLAB software (ode-45). Let us rewrite the model equations in the form of state equations. To do this, it is necessary to denote new symbols as shown:

Let:

$$
\begin{aligned}
& x_{1}=x \quad, \quad x_{2}=y \quad, \quad x_{3}=\theta_{b 1} \quad, \quad x_{2+i}=\theta_{b i} \quad \ldots . x_{2+n}=\theta_{b n} \text {, } \\
& x_{3+n}=\dot{x} \quad x_{4+n}=\dot{y} \quad x_{5}=\dot{\theta}_{b 1} \quad x_{4+n+i}=\dot{\theta}_{b i} \quad x_{4+2 n}=\dot{\theta}_{b n}
\end{aligned}
$$

The equations of motion can be expressed as the state equations which are $(2 n+4)$ first order differential equations. The state equations may be written in a matrix vector equation.

$$
\dot{x}=A(x) x+B
$$

where;

$$
\begin{aligned}
& x=\left[\begin{array}{llllll}
x_{1} & x_{2} & \ldots & x_{2+i} & \ldots \ldots & x_{4+2 n}
\end{array}\right]^{T} \\
& \dot{x}=\left[\begin{array}{llllll}
\dot{x}_{1} & \dot{x}_{2} & \ldots & \dot{x}_{4+n+i} & \ldots \ldots & \dot{x}_{4+2 n}
\end{array}\right]^{T} \\
& A(x)=\left(\begin{array}{cc}
I & 0 \\
0 & M
\end{array}\right)
\end{aligned}
$$

I is $(2+n) \times(2+n)$ identity matrix

$M$ is $(2+n) \times(2+n)$ matrix determined from the above equations,

$B$ is $(4+2 n) \times(4+2 n)$ matrix determined from the model equations.

\section{Validation of the Mathematical Model}

The unbalanced rotor is analytically solved using Jeffcott equations (3-5), also the model is solved numerically with and without the ABB using the above mathematical model. In the numerical simulation, the same model parameters (shown in appendix a) and initial conditions are used. Then, the numerical simulation results are compared to Bolton's results in three cases: the model without balancer, the model with one ball ABB, and the model with two balls ABB.

The rotor vibration amplitudes are calculated at different three values of angular speeds 15 $\mathrm{rad} / \mathrm{s}, 50 \mathrm{rad} / \mathrm{s}$, and $200 \mathrm{rad} / \mathrm{s}$ that are taken by Bolton [8]. The first speed is below the linear spring natural frequencies, the second speed is greater than the linear spring natural frequency and less than the torsional spring natural frequency, and the third one is higher than the torsional spring natural frequency, Table 1 shows the comparison of vibration amplitudes values.

This comparison shows a great agreement between our model results and the results in the reference, the difference between calculated results for rotor amplitude does not exceed $3.7 \%$ which corresponds to $4.5 \times 10^{-7}$ meter, in case of model with one ball ABB at $15 \mathrm{rad} / \mathrm{sec}$. This value can be accepted comparing to the available practical measuring sensitivity. 
Table 1 Comparing values of rotor vibration amplitudes (a) without ABB. (b) with one ball ABB. (c) with two balls ABB.

(a)

\begin{tabular}{|c|c|c|c|}
\hline \multirow{2}{*}{$\begin{array}{c}\text { Angular } \\
\text { speed [rad/s] }\end{array}$} & \multicolumn{3}{|c|}{ Amplitude [m] } \\
\cline { 2 - 3 } & Unbalanced rotor & \multirow{2}{*}{ Reference } \\
\cline { 2 - 3 } & Analytically & Numerically & \\
\hline 15 & $8.4731 \times 10^{-5}$ & $8.4742 \times 10^{-5}$ & $8.582 \times 10^{-5}$ \\
\hline 50 & $2.9993 \times 10^{-4}$ & $2.9988 \times 10^{-4}$ & $3.032 \times 10^{-4}$ \\
\hline 200 & $3.132 \times 10^{-4}$ & $3.1323 \times 10^{-4}$ & $3.1699 \times 10^{-4}$ \\
\hline
\end{tabular}

(b)

\begin{tabular}{|c|c|c|}
\hline \multirow{2}{*}{$\begin{array}{c}\text { Angular } \\
\text { speed [rad/s] }\end{array}$} & \multicolumn{2}{|c|}{ Amplitude [m] } \\
\cline { 2 - 3 } & Model with one ball ABB & Reference \\
\hline 15 & $1.1965 \times 10^{-4}$ & $1.192 \times 10^{-4}$ \\
\hline 50 & $2.8558 \times 10^{-7}$ & 0 \\
\hline 200 & $2.2836 \times 10^{-9}$ & 0 \\
\hline
\end{tabular}

(c)

\begin{tabular}{|c|c|c|}
\hline $\begin{array}{c}\text { Angular } \\
\text { speed [rad/s] }\end{array}$ & \multicolumn{2}{|c|}{ Amplitude [m] } \\
\cline { 2 - 3 } 15 (instable) & $\begin{array}{c}2.008 \times \\
10^{-4}\end{array}$ & Reference \\
\hline 50 & $\begin{array}{c}5.24 \times 10^{-8} \\
\text { no s. } \text { s value can be determined }\end{array}$ \\
\hline 200 & $\begin{array}{c}5.21 \\
\times 10^{-10}\end{array}$ & 0 \\
\hline
\end{tabular}

The ball angular positions are calculated in the two cases model with one ball ABB and with two balls $\mathrm{ABB}$, at the different given values of angular speed $15 \mathrm{rad} / \mathrm{s}, 50 \mathrm{rad} / \mathrm{s}$, and $200 \mathrm{rad} / \mathrm{s}$.

The model with one ball ABB numerical simulation results are compared to Bolton's results [8] as shown in Fig. 4. The continuous curve shows our model simulation and the dotted curves are Bolton's results. A great agreement in the steady state results is shown, but a small deviation in ball dynamic response speed in case of model with one ball ABB. This can be occurred due to imbalance initial angular position [9], and this delay is not interesting in studying the steady state behaviors of the ABB.

Fig. 5.(a) shows the instability of balancing balls at speed of $15 \mathrm{rad} / \mathrm{s}$, this is agreed with the principle of $\mathrm{ABB}$ and this speed below the critical speed out of the balancer stable region. Also, Fig. 5 shows a greet matching of the curves obtained from comparing the numerical solution for the model with two balls ABB and Bolton's results. 


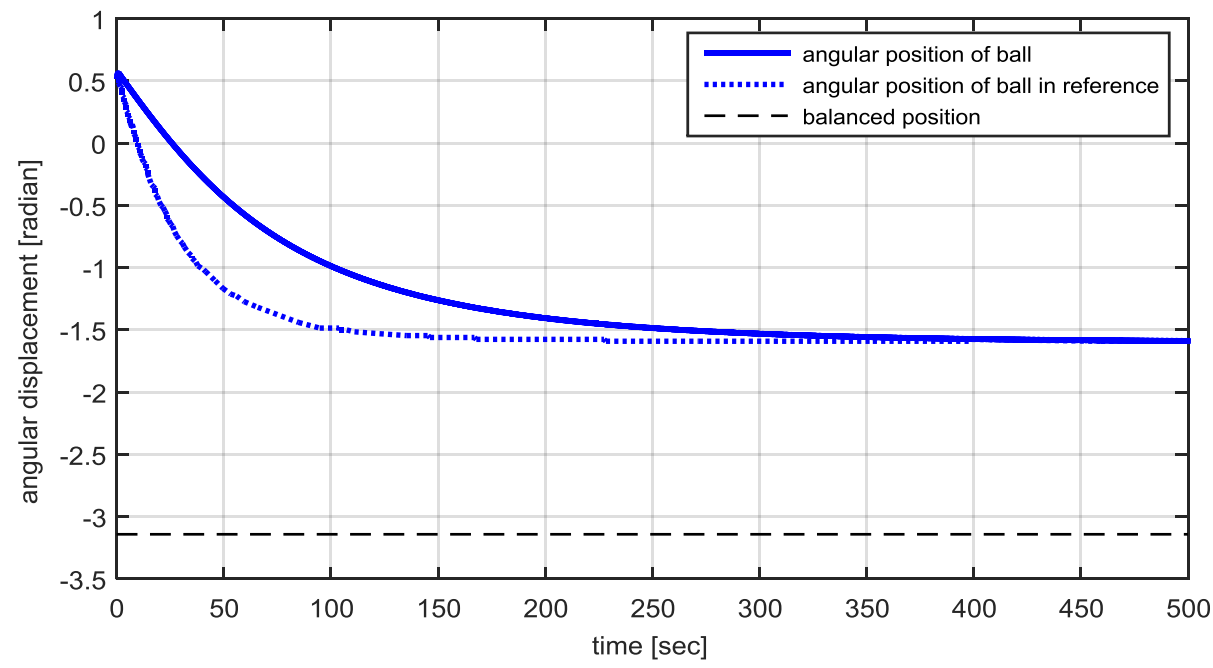

(a)

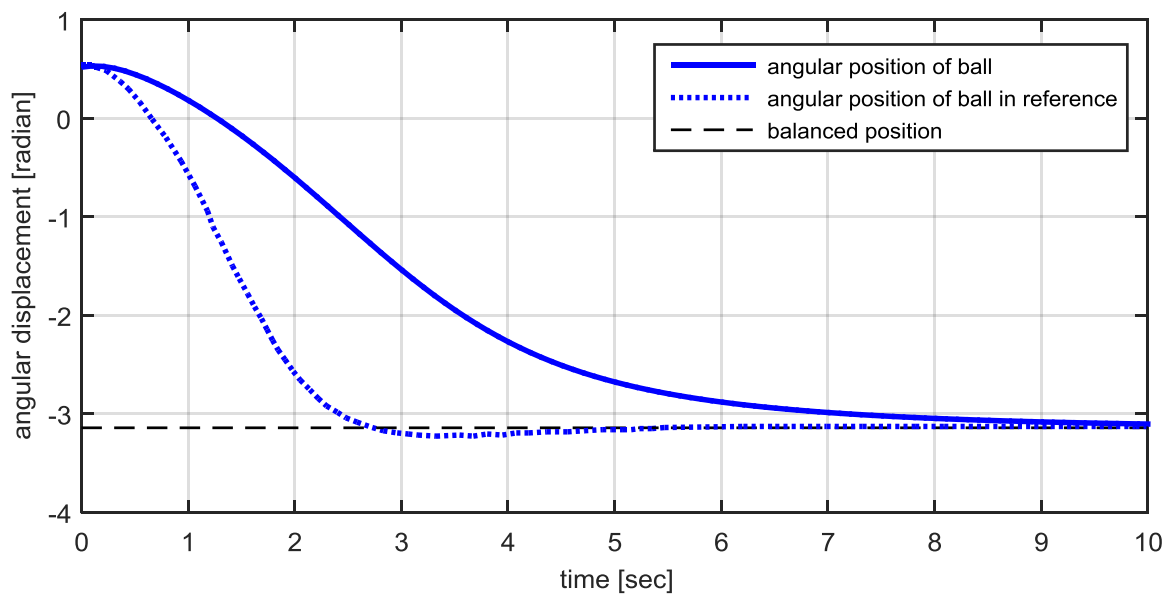

(b)

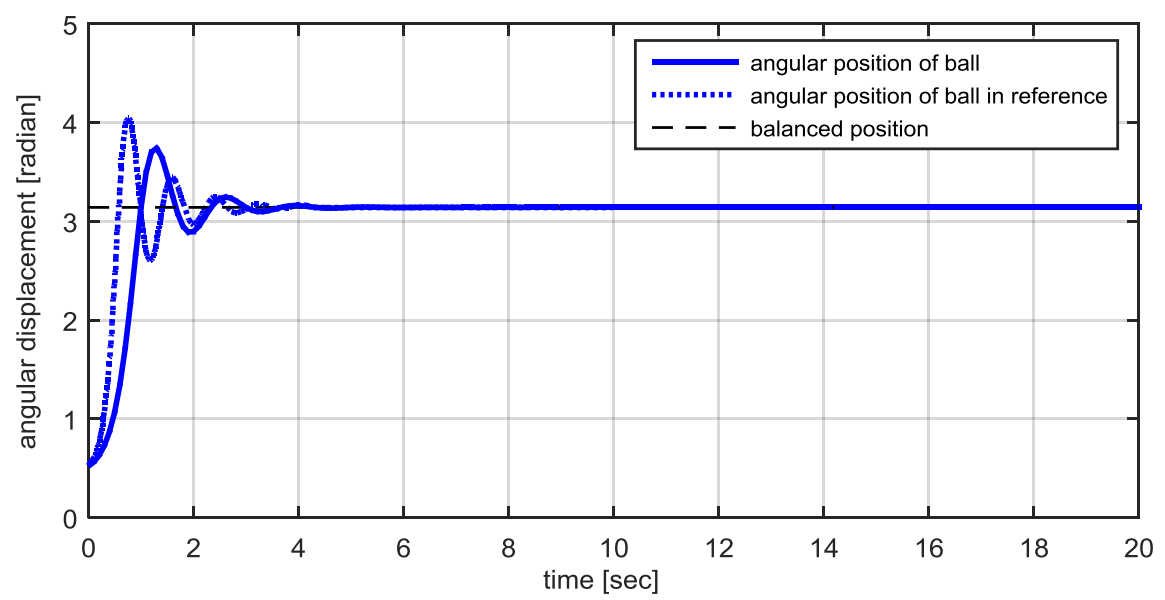

(c)

Fig. 4 Angular position of balancing ball in the model with one ball $\mathrm{ABB}$ at (a) $15 \mathrm{rad} / \mathrm{s}$, (b) $50 \mathrm{rad} / \mathrm{s}$, (c) $200 \mathrm{rad} / \mathrm{s}$. 


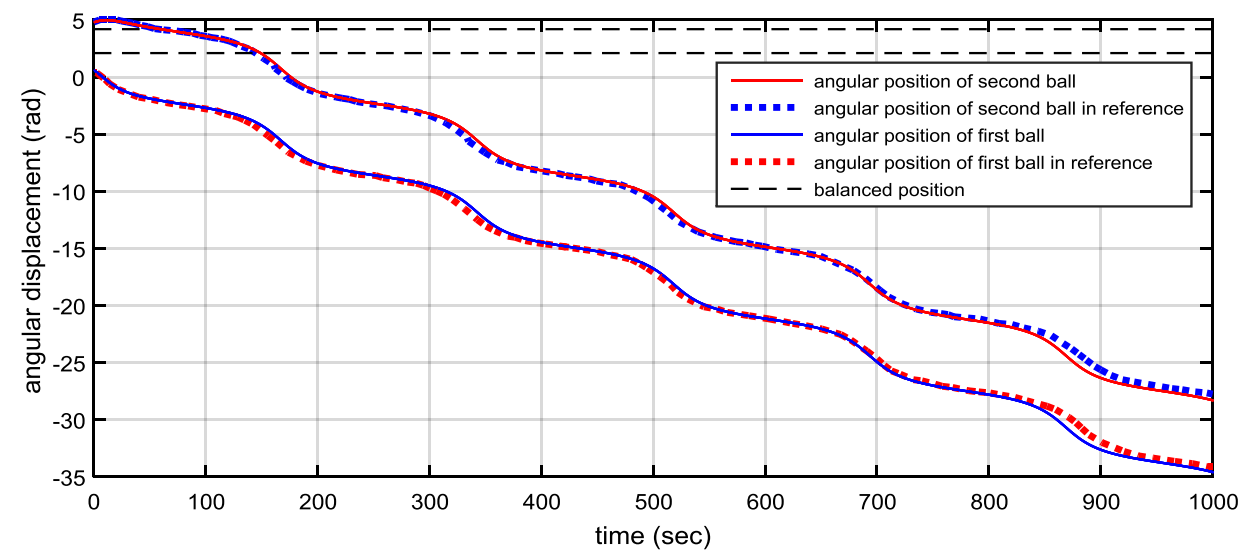

(a)

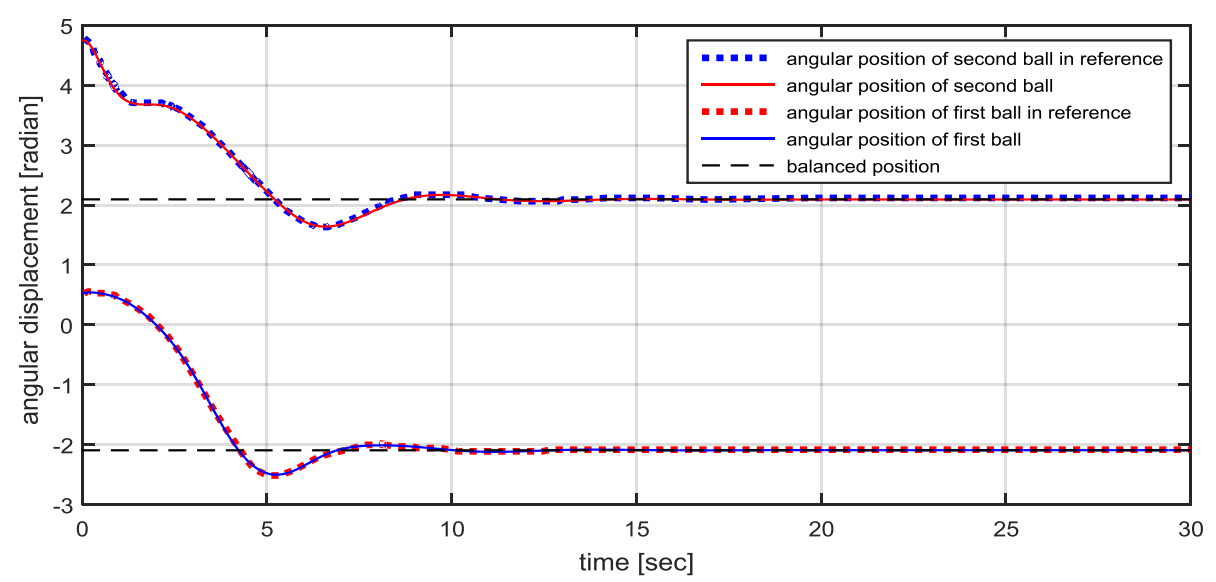

(b)

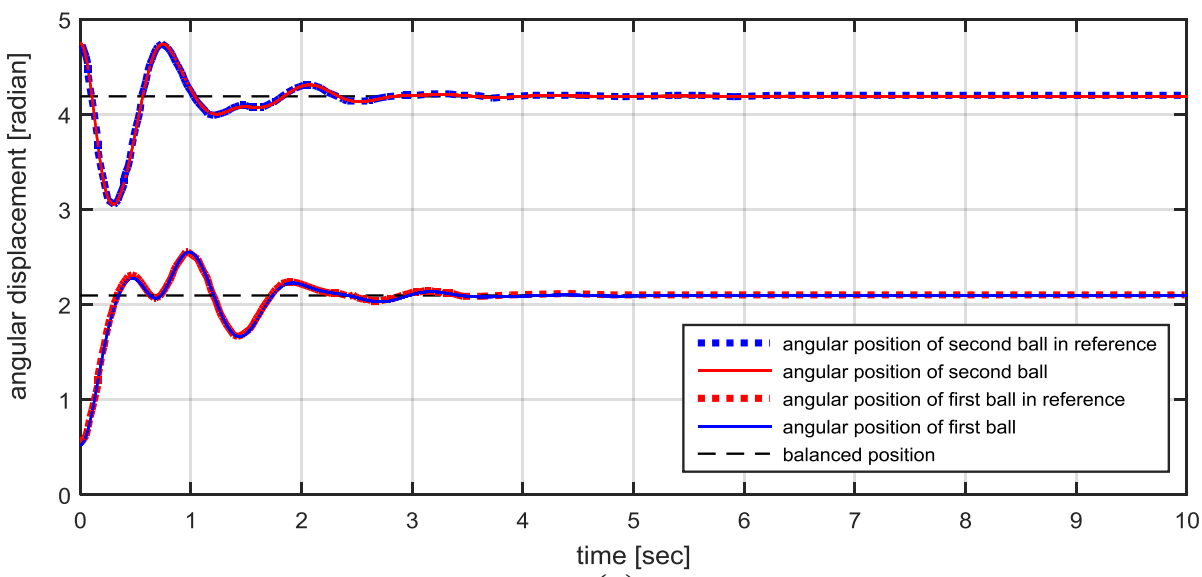

(c)

Fig. 5 Angular position of balancing balls in the model with two balls ABB at (a) $15 \mathrm{rad} / \mathrm{s}$, (b) $50 \mathrm{rad} / \mathrm{s}$, (c) $200 \mathrm{rad} / \mathrm{s}$. 


\section{ABB Effect at Various Speed Ranges}

The ABB effect is clearly shown in Fig. 6 which represents the resonance diagrams of the rotor with and without the balancer across a speed range of 0 to $200 \mathrm{rad} / \mathrm{sec}$. It is shown that the ABB can decrease the vibration amplitudes nearly to zero in angular speeds above the critical speed, but its rule is opposed in speeds below the critical speeds. This is because when the rotor operates at a speed below the natural frequency of the support, the geometric center and the mass imbalance will be on the same side of the disc mass center. While on rotor operating above the natural frequency, an interesting phenomenon occurs, the geometric center and the imbalance are on opposite sides of the disc mass center.

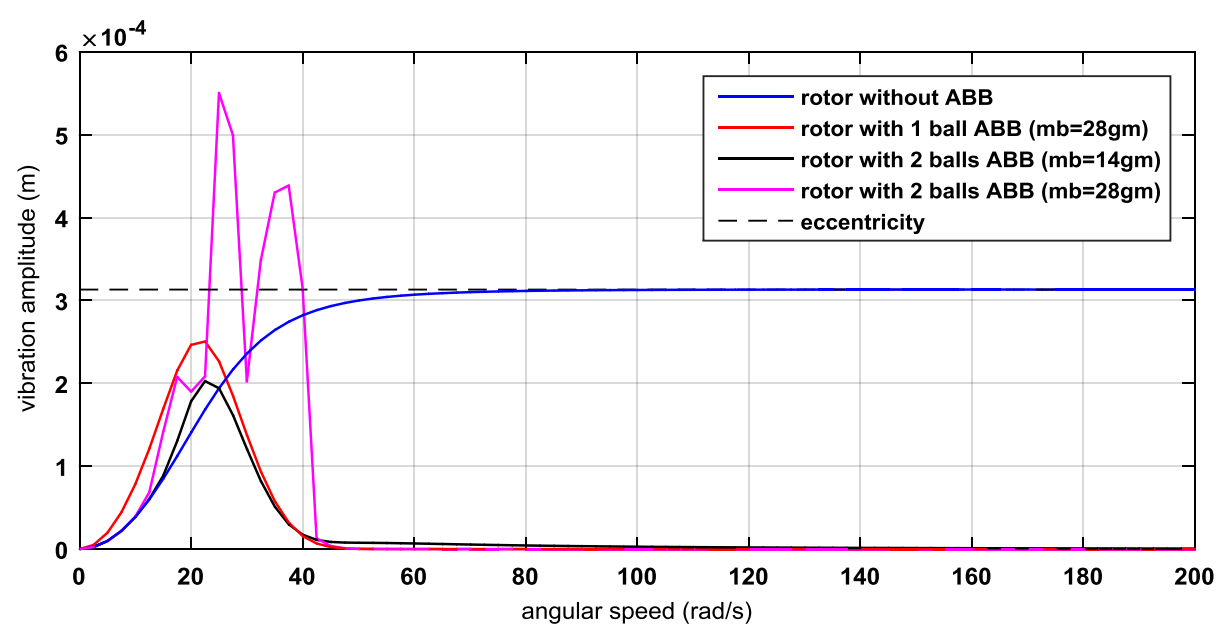

Fig. 6 Resonance diagram

\section{System Damping Coefficient Effect on ABB Behaviors}

It is observed from model simulations that system damping coefficient is one of the most factors affecting ABB rule in damping the vibration results from imbalance. Damping ratio affects the behavior of the unbalanced rotor as known, so that to study its pure effect on $\mathrm{ABB}$, the angular positions of balls are studied in comparison with the wanted balanced position, using different values of damping ratio $(0,0.1,0.3,0.4,0.7,1$, and 1.3$)$ in model simulation at the same three previous speeds.

Fig. 7(a) shows one ball ABB angular position compared to the expected ball balanced position at the speed of $15 \mathrm{rad} / \mathrm{sec}$, it is observed that increasing damping ratio (from 0 to 1.3) bring the balancing ball closer to the balanced position and so improving the balancer behavior, while in Fig. 7(b) one of the balancing balls of the two balls ABB approaches the balanced position and the other goes away from it in the range of damping ratio from 0 to 0.3 , above this damping ratio the balls starts to be instable so that the damping ratio not only affect the steady state position of balancing balls but also it affects clearly the stable range of the ABB. 


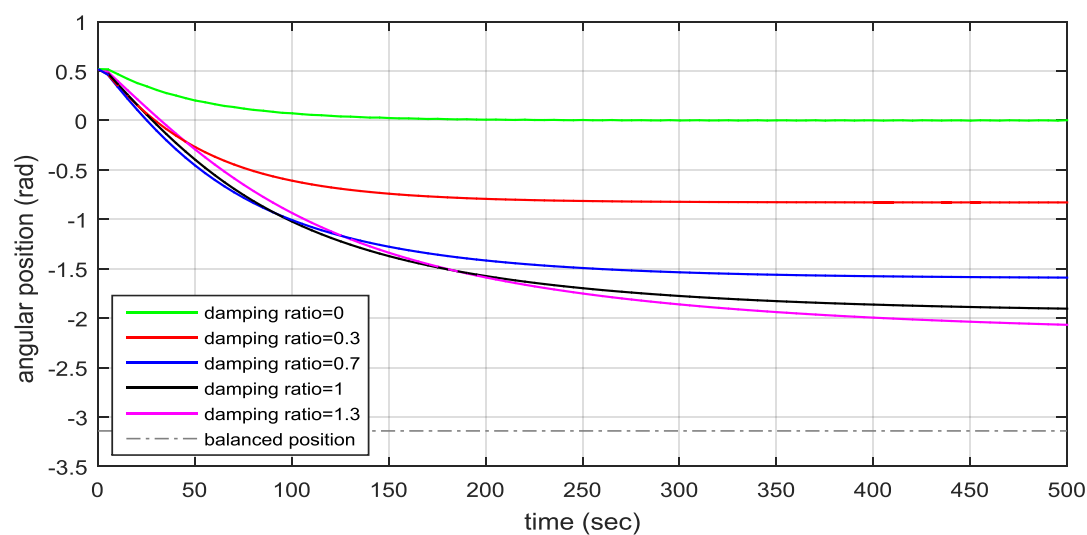

(a)

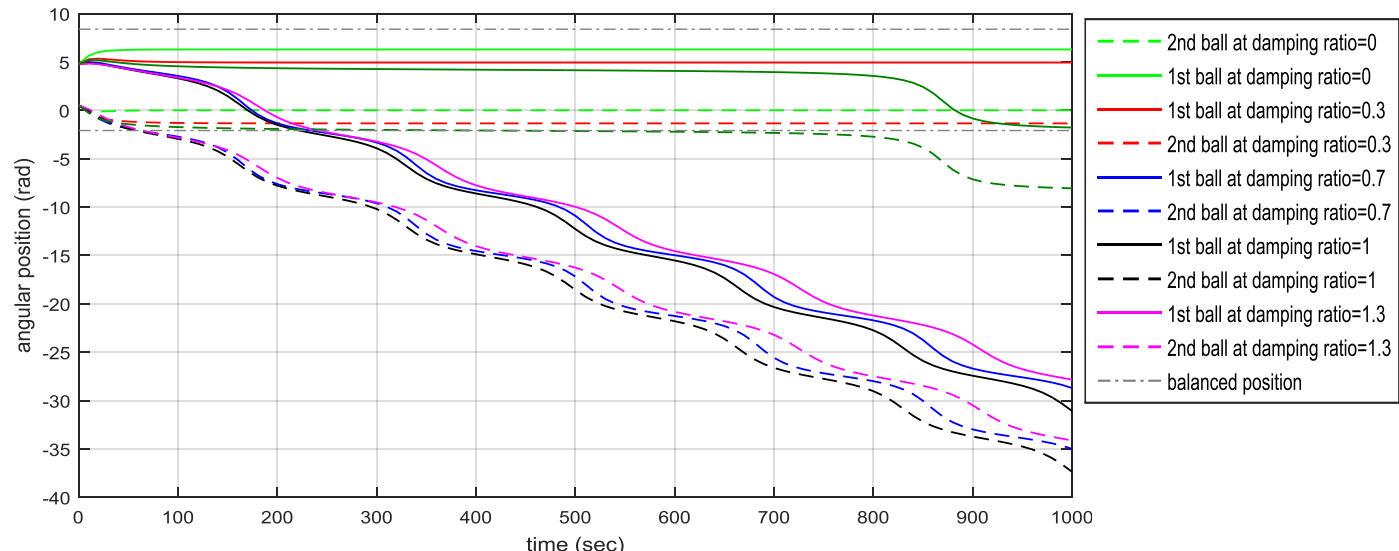

(b)

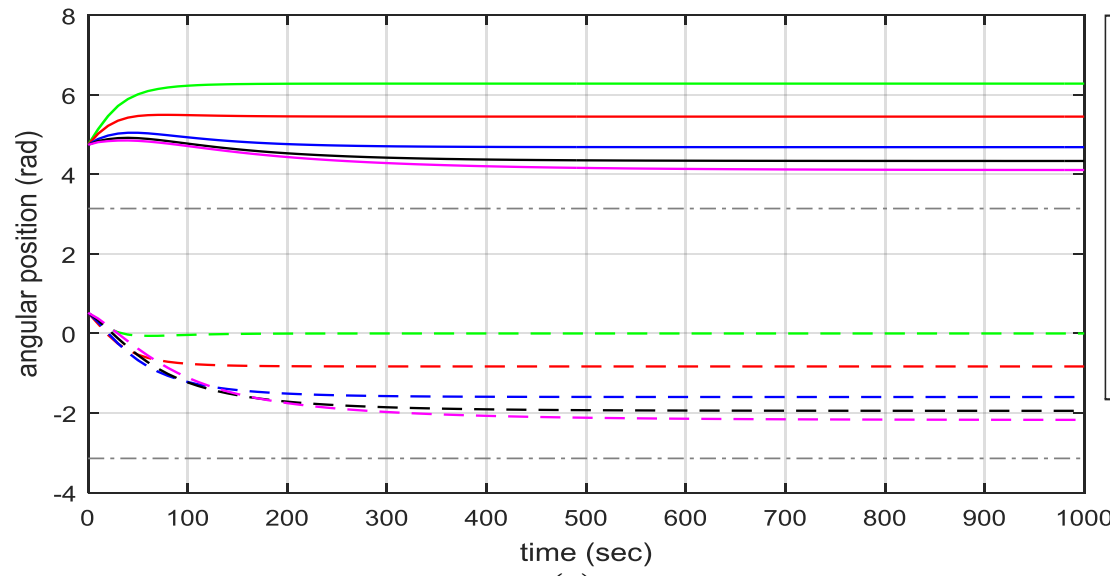

\begin{tabular}{l}
--- 2nd ball at damping ratio $=0$ \\
-1 st ball at damping ratio $=0$ \\
-1 st ball at damping ratio $=0.3$ \\
---2 nd ball at damping ratio $=0.3$ \\
-1 st ball at damping ratio $=0.7$ \\
---2 nd ball at damping ratio $=0.7$ \\
-1 st ball at damping ratio $=1$ \\
---2 nd ball at damping ratio $=1$ \\
-1 st ball at damping ratio $=1.3$ \\
---- 2nd ball at damping ratio $=1.3$ \\
\hline- balanced position
\end{tabular}

(c)

Fig. 7 Angular position of ABB balls at $15 \mathrm{rad} / \mathrm{sec}$.

(a) One ball ABB. (b)Two ball ABB $\left(\mathrm{m}_{\mathrm{b} 1,2}=28 \mathrm{gm}\right)$. (c) Two ball $\mathrm{ABB}\left(\mathrm{m}_{\mathrm{b}}=14 \mathrm{gm}\right)$. 
Balancing ball instability appears at high damping ratio in Fig. 7(b) produced also from bad selection of mass of balancing balls of the two balls ABB. When considering the same balancing masses summation $\left(\mathrm{m}_{\mathrm{b}}=\mathrm{m}_{\mathrm{b} 1}+\mathrm{m}_{\mathrm{b} 2}\right)$, the effect of damping ratio appears purely and it is observed from Fig. 7 (c) that the two balls approach the balanced position and it is shown that it is better to use balls of $14 \mathrm{gm}$ mass in the two balls ABB instead of $28 \mathrm{gm}$ that is used by Bolton [8], so that the total mass of the two balls equal to the mass of the unique balancing ball in one ball $\mathrm{ABB}$ and the comparison between them will be more reasonable.

Fig. 8(a) shows one ball ABB angular position compared to the expected ball balanced position at the speed of $50 \mathrm{rad} / \mathrm{sec}$, it is observed that varying the damping ratio does not affect the balancing ball position in steady state but it delays the dynamic response of the balancing ball impairing the balancer behavior, while in Fig. 8 (b) varying the damping ratio from 0 to 1 has the same effect on the two balancing balls of the ABB , above this damping ratio the balls starts to be instable, using balancing balls of 14 gm mass for the two balls ABB gives the same effect without ball instability.

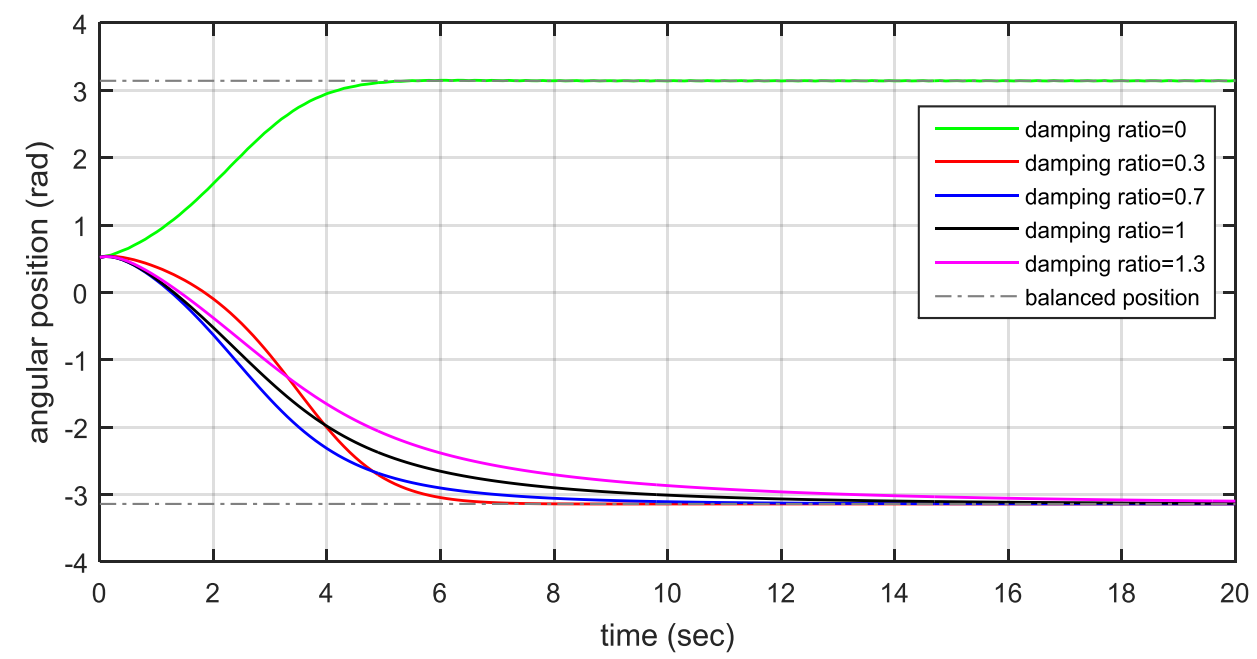

(a)

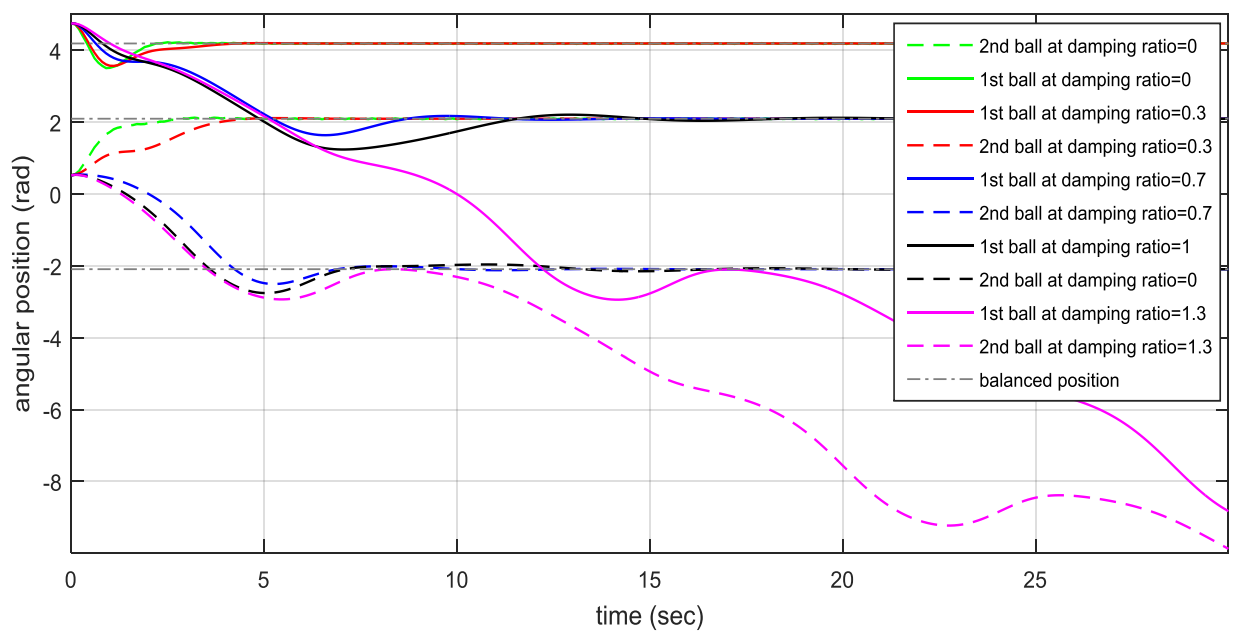

(b)

Fig. 8 Angular position of ABB balls at $50 \mathrm{rad} / \mathrm{sec}$. (a) One ball ABB. (b)Two ball ABB (mb1,2=28gm). 
Fig. 9 shows the angular position of ABB balancing balls compared to the expected ball balanced position at the speed of $200 \mathrm{rad} / \mathrm{sec}$, it is observed that varying the damping ratio does not affect the balancing ball position in steady state but it delays the dynamic response of the balancing ball impairing the balancer behavior, in this speed the balancing balls in the two balls $\mathrm{ABB}$ are stable in the range of damping ratio from 0 to 1.3 so that balls stability in high speeds is lower sensitive to damping ratio variation.

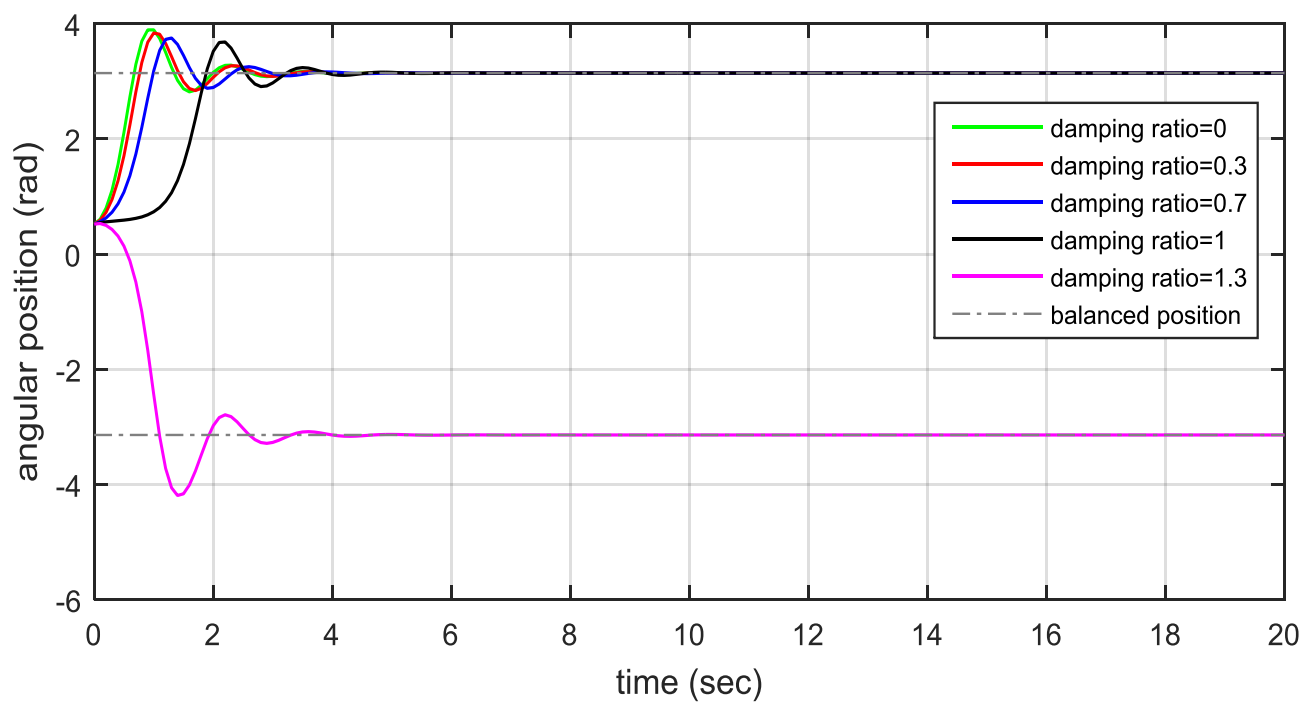

(a)

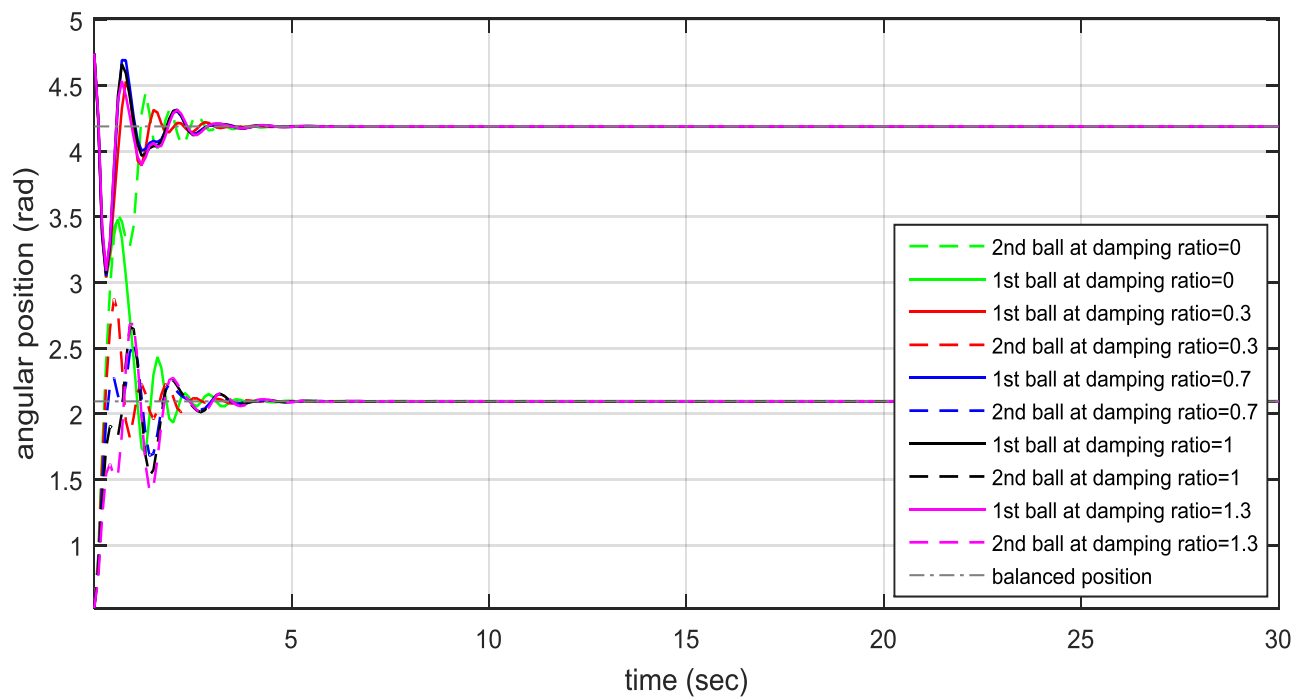

(b)

Fig. 9 Angular position of ABB balls at $200 \mathrm{rad} / \mathrm{sec}$.

(a) One ball ABB. (b)Two ball ABB $\left(\mathrm{m}_{\mathrm{b} 1,2}=28 \mathrm{gm}\right)$. 


\section{Conclusion}

A mathematical model is built up, and solved numerically using MATLAB, to simulate the motion of $\mathrm{ABB}$ balancing balls and the vibration amplitudes of a rotor shaft system with and without ABB. After model validation, the model is trusted to simulate rotor shaft systems with ABB. The ABB main parameters of balancing ball mass, ball damping coefficient, and radial distance of ball track are important in balancer design, but the great relation between the system parameters as damping coefficient of the system and the balancing ball response cannot be ignored. System damping coefficient increase has a good effect on ABB balancing balls motion at low speeds while it delays its responses at high speeds. So, that the ABB is more efficient for damped systems. Summation of ABB balancing balls' masses should be nearly equal or little larger than the expected rotor imbalance, the large difference may disable the balancer from rotor perfect balancing or causes balancing balls instability. Optimization of the balancer parameters taking in consideration system parameters should takes place in the future work, beside the experimental investigation of the $\mathrm{ABB}$ rule in damping the vibration of unbalanced rotor.

\section{References}

[1] Li-fang, C., C. Xi, and G. Jin-Ji, A Study On Electromagnetic Driven Bi-Disc Compensator For Rotor Auto Balancing And Its Movement Control. Wseas Transactions On Systems And Control, 2010. 5( 5).

[2] Fesca, A., Improvement in Centrifugal Machines for Draining Sugar. United States Patent, 1872.

[3] Thearle, E.L., A New Type Of Dynamic-Balancing Machine. Transactions of ASME (applied mechanics), 1932: p. 131-141.

[4] Clark, K., Domestic Appliance. United States Patent, 1940.

[5] Thearle, E.L., Automatic Dynamic Balancers (Part 2-Ring, pendulum, ball balancers). Machine Design, 1950. 22: p. 103-106.

[6] Taekil Kim, S.N., New automatic ball balancer design to reduce transient-response in rotor system. Mechanical Systems and Signal Processing, 2013: p. 265-275.

[7] Hwang, C.H. and J. Chung, Dynamic Analysis of an Automatic Ball Balancer with Double Races. Jsme International Journal, 1999: p. 265-272.

[8] Bolton, J.N., PHD thesis, Single and dual automatic balancing of an elastically cylindrical rotor with considerations of Columb frictions and gravity. 2010.

[9] Chan, T.C., C.K. Sung, and P.C.P. Chao, Non-linear suspension of an automatic ball balancer. International journal of non-linear michanisms (ELSEVIER), 2011: p. 423. 


\section{Appendix (a)}

System parameters used in numerical simulation

\begin{tabular}{|c|c|c|c|}
\hline \multicolumn{2}{|l|}{ parameter } & \multicolumn{2}{|c|}{ value } \\
\hline Rotor mass, & $\mathrm{m}$ & 1.242 slugs & $18.125627 \mathrm{~kg}$ \\
\hline Rotor thickness, & $\mathrm{t}$ & 8 in & $0.2032 \mathrm{~m}$ \\
\hline Rotor inner radias, & $r_{i}$ & 8 in & $0.2032 \mathrm{~m}$ \\
\hline Rotor outer radias, & $r_{o}$ & 9 in & $0.2286 \mathrm{~m}$ \\
\hline Imbalance mass & & 0.0019 slugs & $0.0283268 \mathrm{~kg}$ \\
\hline Imbalance radial distance & & 8 in & $0.2032 \mathrm{~m}$ \\
\hline Imbalance moment & $M_{i m}$ & $0.0155 \mathrm{lb} . \mathrm{ft}$ & 0.005756 N.m \\
\hline Eccentricity, & $\mathrm{e}$ & 0.0125 in & $0.0003176 \mathrm{~m}$ \\
\hline Angular position of imbalance & & $0 \mathrm{deg}$ & $0 \mathrm{rad}$ \\
\hline Gravity acceleration, & $\mathrm{g}$ & $32.2 \mathrm{ft} / \mathrm{sec} 2$ & $9.81 \mathrm{~m} / \mathrm{s} 2$ \\
\hline Stiffness in $\mathrm{x}$-direction, & $k_{x}$ & $1000 \mathrm{lb} / \mathrm{ft}$ & $14632.309 \mathrm{~N} / \mathrm{m}$ \\
\hline Stiffness in y-direction, & $k_{y}$ & $1000 \mathrm{lb} / \mathrm{ft}$ & $14632.309 \mathrm{~N} / \mathrm{m}$ \\
\hline Stiffness in z-direction, & $k_{z}$ & $100000 \mathrm{lb} / \mathrm{ft}$ & $1463230.9 \mathrm{~N} / \mathrm{m}$ \\
\hline Damping coefficientin $\mathrm{x}$-dir., & $c_{x}$ & $49.3 \mathrm{lb} . \mathrm{sec} / \mathrm{ft}$ & 721.37284 N.sec/m \\
\hline Damping coefficientin y-dir., & $c_{y}$ & $49.3 \mathrm{lb} . \mathrm{sec} / \mathrm{ft}$ & 721.37284 N.sec/m \\
\hline Damping coefficientin z-dir., & $c_{z}$ & $500 \mathrm{lb} . \mathrm{sec} / \mathrm{ft}$ & 7316.1546 N.sec $/ \mathrm{m}$ \\
\hline Viscous damping coefficent, & $c_{b}$ & $0.005 \mathrm{lb} . \mathrm{sec} / \mathrm{ft}$ & 0.0731615 N.sec $/ \mathrm{m}$ \\
\hline Balancing ball mass, & $m_{b}$ & 0.0019 slugs & $0.0283122 \mathrm{~kg}$ \\
\hline Radias of ball track, & $a_{b}$ & 8 in & $0.2032 \mathrm{~m}$ \\
\hline
\end{tabular}

Initial conditions used in numerical simulation

\begin{tabular}{|lr|c|c|}
\hline \multicolumn{2}{|c|}{ initial condition } & \multicolumn{2}{c|}{ value } \\
\hline First balancing ball's position, & $\boldsymbol{\theta}_{1}$ & 30 degree & 0.52 radian \\
\hline Second balancing ball's position, & $\boldsymbol{\theta}_{2}$ & 272 degree & $4.75 \mathrm{radian}$ \\
\hline Balls' angular velocity & $\dot{\boldsymbol{\theta}}_{1}, \dot{\boldsymbol{\theta}}_{2}$ & 0 & $0 \mathrm{rad} / \mathrm{sec}$ \\
\hline
\end{tabular}

